\title{
Improved identification of thrombolysis candidates amongst intermediate-risk pulmonary embolism patients: implications for future trials
}

To the Editor:

Contemporary classification of acute pulmonary embolism (PE) severity is based on the risk of early death, which is influenced by demographic factors, comorbidity, and the functional status of the right ventricle (RV) under acute pressure overload [1]. Shock or persistent arterial hypotension, indicating overt RV failure at presentation, has long been identified as a key determinant of poor prognosis [2] and represents the only widely accepted indication for (systemic) thrombolytic therapy to date [3]. In contrast, for normotensive patients who present with imaging findings that indicate RV dysfunction and biochemical evidence of myocardial injury, anticoagulation remains the primary treatment option [3, 4]. This recommendation is supported by the Pulmonary Embolism Thrombolysis (PEITHO) trial, which showed that patients fulfilling these latter criteria were unlikely to derive a net clinical benefit from routine use of systemic thrombolysis in view of the high risk for major bleeding [5].

Although the results of the PEITHO trial are considered as strong evidence against routine full-dose intravenous thrombolysis, they have not terminated the debate on the need for reperfusion treatment of intermediate-risk PE. Outside the setting of randomised controlled trials, these patients continue to have a poor prognosis in the acute phase [6]. Reduced-dose intravenous thrombolysis [7, 8] and pharmaco-mechanical catheter-directed reperfusion techniques $[9,10]$ have emerged as promising options with an improved risk-benefit ratio. In fact, preliminary data from small cohort and phase 2 studies (reviewed in [1]) suggest that these modalities could relieve pulmonary vascular obstruction and improve RV function with a very low rate of life-threatening, particularly intracranial, bleeding complications. It remains to be shown, however, whether their efficacy is comparable, i.e. non-inferior, to that of standard-dose systemic thrombolysis.

The PEITHO trial included normotensive adult patients with objectively confirmed acute PE, RV dysfunction detected on echocardiography or computed tomographic pulmonary angiography, and a positive laboratory test for cardiac troponin I or T. Patients were randomised to receive either a single weight-based intravenous bolus of the fibrinolytic agent tenecteplase, or placebo, with unfractionated heparin. The primary efficacy outcome was the clinical composite of death from any cause or haemodynamic decompensation (or collapse) within 7 days after randomisation [11]. We performed a post hoc analysis of the trial results, focusing on two key aspects, which in our opinion will be crucial for designing future trials that would test safer reperfusion agent dosages or interventions.

First, it would be necessary to identify a higher-risk population, compared to the patients included in PEITHO, as potential candidates for thrombolysis. In fact, PEITHO, which was designed before clinical scores of PE severity were developed and validated, demanded no clinical criteria of 'severe' PE. This limitation may have been responsible, at least in part, for the low absolute rate of the combined primary outcome, and particularly for its component '7-day mortality' in the placebo arm [5]. This, in turn, probably diminished the magnitude of the clinical benefit that reperfusion treatment might have had in a truly 'intermediate-high-risk' population. included in PEITHO http://ow.ly/JM7u30hcSgN

Cite this article as: Barco S, Vicaut E, Klok FA, et al. Improved identification of thrombolysis candidates amongst intermediate-risk pulmonary embolism patients: implications for future trials. Eur Respir J 2018; 51: 1701775 [https://doi.org/10.1183/13993003.01775-2017]. 
TABLE 1 Clinical outcomes of tenecteplase and placebo groups

\begin{tabular}{|c|c|c|c|c|c|c|c|c|c|}
\hline \multicolumn{5}{|c|}{ Placebo (n=499) } & \multicolumn{5}{|c|}{ Tenecteplase ( $n=506$ ) } \\
\hline \multirow{2}{*}{$\begin{array}{l}\text { Criterion } \\
\text { prevalence }\end{array}$} & \multicolumn{2}{|c|}{ Outcome } & \multirow[t]{2}{*}{ p-value } & \multirow[t]{2}{*}{$\operatorname{RR}(95 \% \mathrm{CI})$} & \multirow{2}{*}{$\begin{array}{c}\text { Criterion } \\
\text { prevalence }\end{array}$} & \multicolumn{2}{|c|}{ Outcome } & \multirow[t]{2}{*}{ p-value } & \multirow[t]{2}{*}{$\operatorname{RR}(95 \% \mathrm{CI})$} \\
\hline & $\begin{array}{c}\text { With } \\
\text { criteria }\end{array}$ & $\begin{array}{l}\text { Without } \\
\text { criteria }\end{array}$ & & & & $\begin{array}{l}\text { With } \\
\text { criteria }\end{array}$ & $\begin{array}{l}\text { Without } \\
\text { criteria }\end{array}$ & & \\
\hline
\end{tabular}

\begin{tabular}{|c|c|c|c|c|c|c|c|c|c|c|}
\hline \multicolumn{11}{|l|}{ Clinical severity criteria } \\
\hline $\mathrm{SBP} \leqslant 110 \mathrm{mmHg}$ & $82(16.6)$ & $9(11.0)$ & $24(5.8)$ & 0.087 & $1.89(0.91-3.91)$ & 82 (16.3) & $4(4.9)$ & $15(3.6)$ & 0.530 & $1.37(0.47-4.02)$ \\
\hline $\begin{array}{l}\text { Respiratory rate } \\
>20 \text { breaths } \cdot \min ^{-1}\end{array}$ & $181(46.2)$ & $21(11.6)$ & $6(2.8)$ & $<0.001$ & $4.08(1.68-9.89)$ & $211(51.3)$ & $8(3.8)$ & $4(2.0)$ & 0.281 & $1.90(0.58-6.20)$ \\
\hline Chronic heart failure & $26(5.3)$ & $6(23.1)$ & $26(5.6)$ & 0.004 & $4.14(1.87-9.16)$ & $21(4.2)$ & $1(4.8)$ & $18(3.7)$ & 0.563 & $1.27(0.18-9.07)$ \\
\hline Active cancer & $32(6.7)$ & 6 (18.7) & $26(5.8)$ & 0.014 & $3.22(1.43-7.26)$ & $41(8.4)$ & $3(7.3)$ & $14(3.1)$ & 0.165 & $2.33(0.70-7.76)$ \\
\hline \multicolumn{11}{|c|}{ Model based on bivariate screening only } \\
\hline $\begin{array}{l}\text { At least one severity } \\
\text { criterion }\end{array}$ & $250(50.2)$ & $27(10.8)$ & $6(2.4)$ & $<0.001$ & $4.46(1.88-0.62)$ & $286(56.5)$ & $11(3.8)$ & $8(3.6)$ & 0.902 & $1.06(0.43-2.58)$ \\
\hline At least two criteria & $64(12.9)$ & $13(20.3)$ & $20(4.6)$ & $<0.001$ & $4.41(2.31-8.42)$ & $66(13.0)$ & $5(7.6)$ & $14(3.2)$ & 0.087 & $2.38(0.89-6.39)$ \\
\hline At least three criteria & $7(1.4)$ & $2(28.6)$ & 31 (6.3) & 0.073 & $4.53(1.34-15.33)$ & $2(0.4)$ & $0(0.0)$ & $19(3.8)$ & 1.000 & \\
\hline \multicolumn{11}{|c|}{ Final model based on bivariate screening and clinical judgement ${ }^{+}$} \\
\hline $\begin{array}{l}\text { At least one severity } \\
\text { criterion }^{\S}\end{array}$ & $242(48.6)$ & $27(11.2)$ & $6(2.3)$ & $<0.001$ & $4.76(2.00-11.33)$ & $270(53.5)$ & $10(3.7)$ & $9(3.8)$ & 0.941 & $0.97(0.40-2.34)$ \\
\hline At least two criteria ${ }^{f}$ & $45(9.0)$ & $9(20.0)$ & $24(5.3)$ & 0.001 & $3.78(1.87-7.62)$ & $43(8.5)$ & $3(7.0)$ & $16(3.5)$ & 0.214 & $2.01(0.61-6.64)$ \\
\hline Three criteria & $2(0.4)$ & $0(0.0)$ & $33(6.7)$ & 1.000 & & $1(0.2)$ & $0(0.0)$ & $19(3.8)$ & 1.000 & \\
\hline
\end{tabular}

Data are presented as $\mathrm{n}(\%)$, unless otherwise stated SBP: systolic blood pressure ${ }^{\#}$ : given as the percentage of patients in each treatment arm, in whom the respective variable had been assessed and was available; ${ }^{\uparrow}$ : cancer in model; ${ }^{+}$: cancer not in model; ${ }^{\S}$ : two-tailed $p$-value for interaction $0.011 ;{ }^{f}$ : two-tailed $p$-value for interaction 0.371 .

Second, we believe that it would be important to prospectively define and test, in a future trial, a combined PE-relevant clinical outcome, assessed over a longer period of observation (typically, 30 days rather than only 7 days for the primary outcome of PEITHO). Such an extended outcome will be more clinically relevant, and can be more practicable for calculating the sample size necessary to enable an objective assessment of treatment efficacy.

Based on the above rationale, and seeking to provide useful tools for clinical trials that will test contemporary reperfusion options, we re-analysed the PEITHO data after (retrospectively) defining a combined clinical outcome as the composite of 1) death from any cause; or 2) non-fatal haemodynamic collapse; or 3) non-fatal symptomatic, objectively confirmed recurrent PE, between randomisation and day 30. All events related to this outcome had already been adjudicated by the trial's independent critical events committee [5]. In parallel, we selected baseline clinical criteria of PE severity from the PEITHO dataset, based on their clinical relevance and inclusion in established risk assessment scores (reviewed in [3]). Baseline variables, which were available from the case report forms, and had been listed in the original PEITHO publication [5], were tested for association with the 'new' composite outcome. The optimal cut-off values for continuous variables were chosen based on criteria of clinical relevance, while also taking into account the median values and ranges observed in the PEITHO population. Because of this, some of these values were not identical with the cut-off values used in severity scores, which were developed after PEITHO and focused on much broader study populations of normotensive acute PE patients [1]. The proportion of missing values for the baseline variables included in the present analysis showed no difference between patients randomised to tenecteplase and those who received the placebo (6.1\% versus $6.9 \% ; \mathrm{p}=0.28)$.

Using a two-sided Chi-squared test of proportions, we selected variables that were statistically associated with the outcome at a threshold p-value of 0.1 in the placebo (heparin-only) arm. The selected variables were subsequently tested in the tenecteplase arm, in order to study potential interaction effects. The corresponding relative risk, absolute risk reductions, and 95\% confidence intervals were calculated. The final model was built based on both marginal statistical significance at bivariate screening and external clinical judgement. Data analysis was performed using the SAS software version 9.2 (SAS Institute, Cary, NC, USA).

The composite clinical outcome of death from any cause, non-fatal haemodynamic collapse, or non-fatal symptomatic recurrent PE between randomisation and day 30, occurred in 19 out of 506 (3.8\%) patients in the tenecteplase arm, and in 33 out of $499(6.6 \%)$ in the placebo arm (RR for tenecteplase treatment $0.57,95 \%$ CI $0.33-0.98)$. The results for each treatment arm separately are summarised in table 1. 
Systolic blood pressure $\leqslant 110 \mathrm{mmHg}$, respiratory rate $>20$ breaths $\cdot \mathrm{min}^{-1}$, and chronic heart failure were associated with a significantly higher risk of the PE-focused clinical outcome and were included in the final model. Of 1005 patients randomised in the PEITHO trial [5], 512 (50.9\%) presented with at least one of these three criteria, and $88(8.8 \%)$ presented with at least two (table 1$)$. In the placebo arm, the relative risk for the composite clinical outcome for patients presenting with at least one, or at least two clinical criteria of severity was 4.76 (versus no criterion) and 3.78, respectively (versus one or no criterion). By comparison, the elevation of risk was much less pronounced in the group treated with the thrombolytic agent tenecteplase (relative risk 0.97 and 2.01 for patients with at least one, and at least two criteria of severity, respectively; table 1). The p-values for interaction with treatment were 0.011 and 0.371 , for patients with at least one, and at least two criteria of severity, respectively.

Our study is based on the unique dataset of a large randomised controlled (and thus, free from confounding by indication) thrombolysis trial with adjudicated outcomes, including those defined post hoc for the present analysis. We acknowledge that the data provided in this report are insufficient to establish definitive clinical criteria to identify 'more appropriate' candidates for thrombolysis among normotensive patients with acute PE. Apart from the fact that this was a post hoc analysis, its most important limitation is related to the fact that some potentially relevant baseline parameters, such as syncope, had not been documented in the original case report forms of PEITHO, and it was no longer possible to obtain them retrospectively. For the same reason, we were not able to calculate and directly test more recently developed clinical severity scores (reviewed in [1,3]). Nevertheless, we believe that our results could help to generate clinically relevant hypotheses and provide the background for the design of future reperfusion trials. Our findings support the requirement for clinical indicators of severity at presentation, namely systolic blood pressure $\leqslant 110 \mathrm{mmHg}$, respiratory rate $>20$ breaths $\cdot \mathrm{min}^{-1}$ (or, as a surrogate, hypoxaemia on room air), or chronic heart failure, in addition to established imaging and biochemical criteria, such as those used in PEITHO, to be used as inclusion criteria. Although including patients with at least two criteria might be able to show a greater absolute risk reduction as a result of the therapeutic intervention, these patients comprised only $9 \%$ of the PEITHO population, and this low proportion would be an obstacle to the feasibility of a future trial. Because of this, the requirement of at least one criterion of severity appears to be an adequate and more realistic option, especially when a PE-related composite outcome is chosen.

Stefano Barco $\oplus^{1}$, Eric Vicaut ${ }^{2}$, Frederikus A. Klok $^{1}$, Mareike Lankeit ${ }^{1}$, Guy Meyer ${ }^{3,4,5}$, Stavros V. Konstantinides ${ }^{1,6}$, on behalf of the PEITHO Investigators

${ }^{1}$ Center for Thrombosis and Hemostasis, University Hospital of the Johannes Gutenberg University Mainz, Mainz, Germany. ${ }^{2}$ Clinical Research Unit, Fernand-Widal Hospital, Assistance Publique Hôpitaux de Paris, University Paris Diderot, Paris, France. ${ }^{3}$ Dept of Respiratory Diseases, Hôpital Européen Georges Pompidou, Assistance Publique Hôpitaux de Paris, Paris, France. ${ }^{4}$ Université Paris Descartes, Sorbonne Paris Cité, Paris, France. ${ }^{5}$ INSERM UMRS 970 and CIC 1418, Paris, France. ${ }^{6}$ Cardiology Dept, Democritus University of Thrace, Alexandroupolis, Greece.

Correspondence: Stavros V. Konstantinides, Center for Thrombosis and Hemostasis, University Medical Center Mainz, Langenbeckstrasse 1, 55131 Mainz, Germany. E-mail: stavros.konstantinides@unimedizin-mainz.de

Received: Aug 302017 | Accepted after revision: Oct 312017

Support statement: This work was supported by: the Federal Ministry of Education and Research (BMBF; 01KG0802, $01 \mathrm{EO} 1003$ and 01EO1503) in Germany; the Programme Hospitalier de Recherche Clinique (PHRC; AOM 03063, AOM 08231 and AOM 10171) in France; and a grant from the market authorisation holder of tenecteplase, Boehringer Ingelheim, to the trial sponsor, Assistance Publique Hôpitaux de Paris. The authors are responsible for the contents of this publication. Funding information for this article has been deposited with Crossref Funder Registry.

Conflict of interest: Disclosures can be found alongside this article at erj.ersjournals.com

\section{References}

1 Konstantinides SV, Barco S, Lankeit M, et al. Management of pulmonary embolism: an update. J Am Coll Cardiol 2016; 67: 976-990.

2 Kasper W, Konstantinides S, Geibel A, et al. Management strategies and determinants of outcome in acute major pulmonary embolism: results of a multicenter registry. J Am Coll Cardiol 1997; 30: 1165-1171.

3 Konstantinides SV, Torbicki A, Agnelli G, et al. 2014 ESC Guidelines on the diagnosis and management of acute pulmonary embolism: the Task Force for the Diagnosis and Management of Acute Pulmonary Embolism of the European Society of Cardiology (ESC) Endorsed by the European Respiratory Society (ERS). Eur Heart J 2014; 35: 3033-3073.

4 Kearon C, Akl EA, Ornelas J, et al. Antithrombotic therapy for VTE disease: CHEST Guideline and Expert Panel Report. Chest 2016; 149: 315-352.

5 Meyer G, Vicaut E, Danays T, et al. Fibrinolysis for patients with intermediate-risk pulmonary embolism. $N$ Engl J Med 2014; 370: 1402-1411.

6 Becattini C, Agnelli G, Lankeit M, et al. Acute pulmonary embolism: mortality prediction by the 2014 European Society of Cardiology risk stratification model. Eur Respir J 2016; 48: 780-786. 
7 Sharifi M, Bay C, Skrocki L, et al. Moderate pulmonary embolism treated with thrombolysis (from the "MOPETT" Trial). Am J Cardiol 2013; 111: 273-277.

8 Wang C, Zhai Z, Yang Y, et al. Efficacy and safety of low dose recombinant tissue-type plasminogen activator for the treatment of acute pulmonary thromboembolism: a randomized, multicenter, controlled trial. Chest 2010; 137: 254-262.

9 Kucher N, Boekstegers P, Muller OJ, et al. Randomized, controlled trial of ultrasound-assisted catheter-directed thrombolysis for acute intermediate-risk pulmonary embolism. Circulation 2014; 129: 479-486.

10 Piazza G, Hohlfelder B, Jaff MR, et al. A prospective, single-arm, multicenter trial of ultrasound-facilitated, catheter-directed, low-dose fibrinolysis for acute massive and submassive pulmonary embolism: the SEATTLE II Study. JACC Cardiovasc Interv 2015; 8: 1382-1392.

11 Single-bolus tenecteplase plus heparin compared with heparin alone for normotensive patients with acute pulmonary embolism who have evidence of right ventricular dysfunction and myocardial injury: rationale and design of the Pulmonary Embolism Thrombolysis (PEITHO) trial. Am Heart J 2012; 163: 33-38.

Copyright @ERS 2018 